\title{
A REMARK ON PARTIAL RESOLUTIONS OF 3-DIMENSIONAL TERMINAL SINGULARITIES
}

\author{
TAKAYUKI HAYAKAWA
}

\begin{abstract}
Let $X$ be a 3 -dimensional terminal singularity of index $\geq 2$. We study projective birational morphisms $\varphi: Y \rightarrow X$ such that the exceptional divisor of $\varphi$ consists of all prime divisors with discrepancies $<1$ (resp. $\leq 1)$ over $X$.
\end{abstract}

\section{$\S 1$. Introduction}

In this note, we shall study a problem on birational morphisms of 3folds which is proposed by [Reid87, (6.5)]. It asks the existence of economic resolutions of 3-dimensional terminal singularities.

Let $P \in X$ be a germ of a 3 -dimensional terminal singularity. Then [Reid87, (6.5)] asks if there is a partial resolution $f: Y \rightarrow X$ such that all exceptional prime divisors have discrepancies $<1$ and $Y$ has only isolated $\mathrm{cDV}$ points. However the condition on singularities of $Y$ seems to be too strong. In fact if such a partial resolution $f: Y \rightarrow X$ exists and if the exceptional set of $f$ is a divisor, then every prime divisor with discrepancy $\leq 1$ over $P(\in X)$ appears as a divisor on $Y$. On the other hand, we saw in [Hay05] that there are prime divisors with discrepancies 1 over $P$ in many cases. Thus we change the condition on singularities of $Y$ and define variants of economic resolutions as follows:

Definition 1.1. A projective birational morphism $f: Y \rightarrow X$ is called a terminal economic (resp. terminal *-economic) resolution if the following conditions (i) and (ii) are satisfied:

(i) The set of all exceptional prime divisors of $f$ is exactly the set of all prime divisors with discrepancies $<1$ (resp. $\leq 1)$ over $P(\in X)$.

(ii) $Y$ has only $\mathbb{Q}$-factorial terminal singularities.

Received January 30, 2003.

Revised July 1, 2004.

2000 Mathematics Subject Classification: 14B05. 
By using results in [Hay05], we can show that $P \in X$ has terminal economic and terminal $*$-economic resolutions in many cases, e.g., $P \in X$ is of type $(\mathrm{cA} / m)$. However there are examples of $P \in X$ which have no such resolutions if $P \in X$ is of type $(\mathrm{cE} / 2)$. The main purpose of this note is to give such examples and prove that there are no terminal economic or terminal $*$-economic resolutions. Our examples of $P \in X$ are $\mathbb{Q}$-factorial so that the exceptional set of each birational morphism to $P \in X$ is a divisor. These will be done in Sections 3 and 4 respectively.

Thus we again have to change the condition on singularities of $Y$, and get to the following:

Definition 1.2. A projective birational morphism $f: Y \rightarrow X$ is called a canonical economic (resp. canonical $*$-economic) resolution if the conditions (i) and (ii)' are satisfied:

(ii)' $Y$ has only $\mathbb{Q}$-factorial canonical singularities.

We can show that such resolutions always exist and proofs will be given in Section 5. We remark that we need not assume $P \in X$ is $\mathbb{Q}$-factorial. These will be done by an application of the Minimal Model Program.

The author would like to thank Professor S. Mori for his invaluable suggestions and encouragement.

\section{$\S 2$. Notation and preliminaries}

2.1. Let $P \in X$ be a germ of a 3 -dimensional terminal singularity. Let $\Delta_{X}$ be a $\mathbb{Q}$-Cartier $\mathbb{Q}$-divisor on $X$ and let $\mathcal{H}_{X}$ be a linear system on $X$ which consists of $\mathbb{Q}$-Cartier divisors.

Let $\mu: \tilde{X} \rightarrow X$ be a resolution of singularities of $X$. We denote the birational transform of $\Delta_{X}\left(\operatorname{resp} . \mathcal{H}_{X}\right)$ by $\Delta_{\tilde{X}}\left(\right.$ resp. $\left.\mathcal{H}_{\tilde{X}}\right)$, and write

$$
\begin{aligned}
& K_{\tilde{X}}+\Delta_{\tilde{X}}=\mu^{*}\left(K_{X}+\Delta_{X}\right)+\sum a\left(E, X, \Delta_{X}\right) E \\
& K_{\tilde{X}}+\mathcal{H}_{\tilde{X}}=\mu^{*}\left(K_{X}+\mathcal{H}_{X}\right)+\sum a\left(E, X, \mathcal{H}_{X}\right) E
\end{aligned}
$$

where the sum in the right hand side runs over all exceptional prime divisors $E$ of $\mu$ and $a\left(E, X, \Delta_{X}\right), a\left(E, X, \mathcal{H}_{X}\right) \in \mathbb{Q}$. The coefficient $a\left(E, X, \Delta_{X}\right)$ (resp. $\left.a\left(E, X, \mathcal{H}_{X}\right)\right)$ of $E$ is called the discrepancy of $E$ over $X$ with respect to $\Delta_{X}$ (resp. $\left.\mathcal{H}_{X}\right)$. This depends only on the discrete valuation on the function field $\mathbb{C}(X)$ of $X$ determined by $E$, and does not depend on the particular resolution $\mu$. Thus $a\left(E, X, \Delta_{X}\right)$ and $a\left(E, X, \mathcal{H}_{X}\right)$ are defined for 
all exceptional prime divisors $E$ over $X$. When $\Delta_{X}=0$, we denote $a(E, X)$ instead of $a\left(E, X, \Delta_{X}\right)$ and call it the discrepancy of $E$ over $X$. We often identify exceptional prime divisors over $X$ with the corresponding discrete valuations.

In this note, we are interested in prime divisors $E$ with $a(E, X)<1$ and $a(E, X) \leq 1$.

In order to calculate discrepancies, we frequently use the following:

Lemma 2.2. Let $P \in X$ and $\Delta_{X}$ be as in (2.1). Let $f: Y \rightarrow X$ be a projective birational morphism such that $Y$ has only terminal singularities. We write $K_{Y}=f^{*} K_{X}+\sum a_{i} E_{i}$, where $\sum E_{i}$ is the exceptional divisor of $f$ and $a_{i} \in \mathbb{Q}$. Then for each exceptional prime divisor $F$ over $Y$, we have

$$
a\left(F, X, \Delta_{X}\right)=a\left(F, Y, f^{*} \Delta_{X}-\sum a_{i} E_{i}\right) .
$$

Proof. Let $g: Z \rightarrow Y$ be a resolution and let $h=f \circ g$. Then we have $h^{*}\left(K_{X}+\Delta_{X}\right)=g^{*}\left(K_{Y}+f^{*} \Delta_{X}-\sum a_{i} E_{i}\right)$. The result is an easy consequence of this equality.

The following two lemmas will also be used in Sections 3 and 4 . The first one is taken from [FA92, 6.2] and the second one from [Kaw84, 5.9].

Lemma 2.3. Let $P \in X$ be as in (2.1) and let $f_{i}: Y_{i} \rightarrow X(i=1,2)$ be projective birational morphisms such that the exceptional set of $f_{i}$ is an irreducible divisor $E_{i}$. If $E_{1}$ and $E_{2}$ define the same valuation on $\mathbb{C}(X)$, then $Y_{1}$ and $Y_{2}$ are isomorphic over $X$.

LEMMA 2.4. Let $f: Y \rightarrow X$ be a projective birational morphism (not an isomorphism) of 3 -folds with only $\mathbb{Q}$-factorial terminal singularities. Then $K_{Y}$ is not $f$-nef.

\section{§3. Terminal economic resolutions}

In this section, we shall give an example of a 3-dimensional terminal singularity which does not have terminal economic resolutions.

3.1. Let $P \in X$ be a germ of a 3 -dimensional $\mathbb{Q}$-factorial terminal singularity, and assume that this is of type $(\mathrm{cE} / 2)$. Then there is an analytic embedding $X \hookrightarrow(x, y, z, u) / \mathbb{Z}_{2}(0,1,1,1)$ such that

$$
X=\left\{u^{2}+x^{3}+g(y, z) x+h(y, z)=0\right\} / \mathbb{Z}_{2}(0,1,1,1),
$$


where $g(y, z), h(y, z) \in(y, z)^{4} \mathbb{C}\{y, z\}$ are $\mathbb{Z}_{2}$-invariants and the degree 4 part $h_{\operatorname{deg} 4}(y, z)$ of $h(y, z)$ is nonzero.

In (3.2) and (3.3), we shall construct birational morphisms $f: Y \rightarrow X$ and $g_{1}: Z_{1} \rightarrow Y$ using this embedding, hence in the analytic category. However, using the algebraization theorems by Artin ([Art68], [Art69]), we have the corresponding morphisms in the algebraic category.

Here we assume that $h_{\operatorname{deg} 4}(y, z)=y^{4}$ and that $\tau-w t(g(y, z)) \geq 4$, $\tau-w t(h(y, z)) \geq 6$ when we set $\tau$-wt $(y)=3 / 2, \tau-w t(z)=1 / 2$. We also assume that the blow up $f: Y \rightarrow X$ with weight $(x, y, z, u)=\frac{1}{2}(4,3,1,7)$ is divisorial with discrepancy $1 / 2$. Thus we are in the situation of [Hay99, 10.59-10.64]. We also remark that prime divisors with discrepancies $<1$ over $X$ always have discrepancies $1 / 2$, and that the center of these divisors is $P$.

Proposition 3.2. Let $P \in X$ be as in (3.1). Then there are exactly two prime divisors with discrepancies $1 / 2$ over $P(\in X)$ and $f: Y \rightarrow X$ in (3.1) is the unique divisorial blow up of $P \in X$ with discrepancy $1 / 2$.

Proof. This follows from [Hay99, 10.61, 10.62].

3.3. Let $P \in X$ and $f: Y \rightarrow X$ be as in (3.1) and let $E$ be the exceptional divisor of $f$. By [Hay99, 10.6, 10.61], $Y$ is Gorenstein outside one point $Q$, and there is an open neighborhood $Q \in U \subseteq Y$ such that $U \simeq$ $(x, y, z) / \mathbb{Z}_{7}(4,3,1)$ with $E_{\mid U}=\operatorname{div}_{U}\left(x^{3}+(g(y, z))_{\tau-w t=4} \cdot x+(h(y, z))_{\tau-w t=6}\right)$. Let $g_{1}: Z_{1} \rightarrow Y$ be the blow up at $Q$ with weight $(x, y, z)=\frac{1}{7}(1,6,2)$ in $(x, y, z) / \mathbb{Z}_{7}(4,3,1)(\simeq U \subseteq Y)$. The exceptional divisor $F_{1}$ of $g_{1}$ is irreducible and we have

$$
a\left(F_{1}, X\right)=a\left(F_{1}, Y,-\frac{1}{2} E\right)=a\left(F_{1}, U,-\frac{1}{2} E_{\mid U}\right)=2 / 7+1 / 2 \cdot 3 / 7=1 / 2
$$

by using (2.2). Let $\varphi_{1}=f \circ g_{1}: Z_{1} \rightarrow X$ be the composition. Then the exceptional set of $\varphi_{1}$ consists of two prime divisors $g_{1 *}^{-1} E$ and $F_{1}$, which have both discrepancies $1 / 2$ over $X$. However $\varphi_{1}$ is not a terminal economic resolution, since $Z_{1}$ has singularities which are not terminal. In fact $Z_{1}$ has 1-dimensional singular locus which are canonical.

Theorem 3.4. Let $P \in X$ be as in (3.1). Then $P \in X$ has no terminal economic resolutions.

Proof. Assuming that there is a terminal economic resolution $\varphi: Z \rightarrow$ $X$, we shall derive a contradiction. The exceptional set of $\varphi$ consists of 
two prime divisors, both of which have discrepancies $1 / 2$ over $X$, hence we know that $\rho(Z / X)=2$. By $(2.4), K_{Z}$ is not $\varphi$-nef, so there is an extremal ray $R$ on $Z$ such that $\left(K_{Z} \cdot R\right)<0$. We apply the Minimal Model Program over $X$ starting with the contraction of $R$. Then, after a finite number of flips $Z \rightarrow Z^{\prime}$, we get to a divisorial contraction $g^{\prime}: Z^{\prime} \rightarrow Y^{\prime}$. We know that both $Z^{\prime}$ and $Y^{\prime}$ have only terminal singularities. Since $\rho\left(Y^{\prime} / X\right)=1$, the exceptional set of $f^{\prime}: Y^{\prime} \rightarrow X$ is a prime divisor which has discrepancy $1 / 2$ over $X$. Since $Y^{\prime}$ has only terminal singularities, it follows from (2.3) and (3.2) that $f^{\prime}: Y^{\prime} \rightarrow X$ is isomorphic to $f: Y \rightarrow X$ in (3.1). By using (2.3) again for $g^{\prime}: Z^{\prime} \rightarrow Y^{\prime}$ and $g_{1}: Z_{1} \rightarrow Y$ in (3.3), we see that $Z^{\prime}$ and $Z_{1}$ are isomorphic over $Y\left(\simeq Y^{\prime}\right)$. This is a contradiction, since $Z^{\prime}$ has only terminal singularities and $Z_{1}$ has singularities worse than terminal.

\section{$\S 4$. Terminal *-economic resolutions}

In this section, we shall give an example of a 3-dimensional terminal singularities which does not have terminal *-economic resolutions. The discussion below are almost the same as in Section 3. We have to work a little more because we shall treat three exceptional prime divisors.

4.1. Let $P \in X$ be a germ of a 3 -dimensional $\mathbb{Q}$-factorial terminal singularity, and assume again that this is of type $(\mathrm{cE} / 2)$. Then $X$ has an analytic embedding as in (3.1.1). We write $g(y, z)=\sum_{p, q} a_{p q} y^{p} z^{q}$ and $h(y, z)=\sum_{p, q} b_{p q} y^{p} z^{q}$ as power series. We again remark that, though our construction of birational morphisms will be done in the analytic category, there are corresponding morphisms also in the algebraic category.

We shall assume that $h_{\operatorname{deg} 4}(y, z)=y^{4}$ and that the system of equations

$$
t^{3}+a_{04} t+b_{06}=0, \quad 3 t^{2}+a_{04}=0, \quad a_{13} t+b_{15}=0
$$

has no solutions in $t$. Then, by [Hay05, 7.8], we see that the blow up $f$ : $Y \rightarrow X$ with weight $(x, y, z, u)=\frac{1}{2}(2,3,1,3)$ is divisorial with discrepancy $1 / 2$. Thus we are in the situation of [Hay05, 7.8(A)].

Proposition 4.2. Let $P \in X$ be as in (4.1). Then there are exactly three prime divisors with discrepancies $\leq 1$ over $P(\in X)$. Among these three divisors, one of these has discrepancy $1 / 2$ and others have discrepancies 1. Furthermore, $f: Y \rightarrow X$ in (4.1) is the unique divisorial blow up at $P$ with discrepancy $\leq 1$. 
Proof. We know the number of prime divisors with discrepancies $\leq 1$ by [Hay05, 7.9]. Thus it suffices to show that there are no divisorial blow ups of $X$ with discrepancies 1 . We start with finding two prime divisors with discrepancies 1 over $P(\in X)$.

Let $f_{1}: Y_{1} \rightarrow X$ be the blow up with weight $(x, y, z, u)=(2,2,1,3)$. Then $Y_{1}$ has an open subset $U_{1}$ (which is the $z$-chart of $Y_{1}$ ) such that

$$
U_{1} \simeq\left\{u^{2}+x^{3}+g\left(y z^{2}, z\right) / z^{4} \cdot x+h\left(y z^{2}, z\right) / z^{6}=0\right\} / \mathbb{Z}_{2}(0,1,1,0),
$$

hence $Y_{1}$ has singularities which are not terminal. The exceptional set $E_{1}$ of $f_{1}$ is an irreducible divisor over $P$ and satisfies $a\left(E_{1}, X\right)=1$. Similarly, let $f_{2}: Y_{2} \rightarrow X$ be the blow up with weight $(x, y, z, u)=(2,1,1,2)$. Then the exceptional set $E_{2}$ of $f_{2}$ is an irreducible divisor over $P$ which satisfies $a\left(E_{2}, X\right)=1$. By studying singularities of $Y_{2}$, we again see that $Y_{2}$ has non terminal singularities. We also remark that $f_{1}$ and $f_{2}$ are not isomorphic over $X$ and that $E_{1}$ and $E_{2}$ are the prime divisors with discrepancies 1 over $P(\in X)$.

Now assume that there is a divisorial blow up $f^{\prime}: Y^{\prime} \rightarrow X$ with discrepancy 1. Then it follows from (2.3) that $Y^{\prime}$ is isomorphic to either $Y_{1}$ or $Y_{2}$. This is a contradiction by comparing singularities of $Y^{\prime}$ with those of $Y_{1}$ (or $Y_{2}$ ). Thus we know that there are no divisorial blow ups of $X$ with discrepancies 1.

4.3. Let $P \in X$ and $f: Y \rightarrow X$ be as in (4.1) and let $E$ be the exceptional divisor of $f$. By [Hay05, 7.2], $Y$ is Gorenstein outside one point $Q$, and there is an open neighborhood $Q \in U \subseteq Y$ such that

$U \simeq\left\{u^{2}+x^{3}+g\left(y^{3 / 2}, y^{1 / 2} z\right) / y^{2} \cdot x+h\left(y^{3 / 2}, y^{1 / 2} z\right) / y^{3}=0\right\} / \mathbb{Z}_{3}(2,1,1,0)$.

This is terminal of type $(\mathrm{cD} / 3)$. We also see that $E_{\mid U}=\operatorname{div}_{U}(y)$.

Let $g: Z \rightarrow Y$ be the blow up at $Q$ with weight $(x, y, z, u)=\frac{1}{3}(2,4,1,3)$ in $U(\subseteq Y)$. Since (4.1.1) has no solutions in $t$, we know that $g$ is divisorial with discrepancy $1 / 3$. Let $F$ be the exceptional divisor of $g$. By using (2.2), we have

$$
a(F, X)=a\left(F, Y,-\frac{1}{2} E\right)=a\left(F, U,-\frac{1}{2} \operatorname{div}_{U}(y)\right)=1 / 3+1 / 2 \cdot 4 / 3=1 .
$$

Thus the composition $\varphi=f \circ g: Z \rightarrow X$ is a projective birational morphism such that the exceptional set consists of two prime divisors $F$ and $g_{*}^{-1} E$. 
We see that $Z$ is Gorenstein outside one point $Q^{\prime}$, and there is an open neighborhood $Q^{\prime} \in U^{\prime} \subseteq Z$ such that

$$
U^{\prime} \simeq\left\{u^{2}+x^{3}+g\left(y^{2}, y z\right) / y^{4} \cdot x+h\left(y^{2}, y z\right) / y^{6}=0\right\} / \mathbb{Z}_{4}(2,1,1,3) .
$$

This is terminal of type $(\mathrm{cAx} / 4)$. We also see that $\left(-\frac{1}{3} F-\frac{1}{2} g^{*} E\right)_{\mid U^{\prime}}=$ $-\operatorname{div}_{U^{\prime}}(y)$.

Lemma 4.4. Let $Y, U, Q$ be as in (4.3). Let $g_{1}: Z_{1} \rightarrow Y$ be the blow up at $Q$ with weight $(x, y, z, u)=\frac{1}{3}(4,2,2,3)$ in $U(\subseteq Y)$. Then the exceptional set $F_{1}$ of $g_{1}$ is an irreducible divisor with $a\left(F_{1}, X\right)=1$, and $Z_{1}$ has non terminal singularities.

Proof. There is an open subset $V_{1} \subseteq Z_{1}$ (which is the $z$-chart of $Z_{1}$ ) such that

$$
V_{1} \simeq\left\{\begin{array}{c}
u^{2}+x^{3} z^{2}+\left(g\left(y^{3 / 2} z, y^{1 / 2} z\right) / y^{2} z^{2}\right) \cdot x \\
+h\left(y^{3 / 2} z, y^{1 / 2} z\right) / y^{3} z^{4}=0
\end{array}\right\} / \mathbb{Z}_{2}(0,0,1,1) .
$$

Thus $Z_{1}$ has non terminal singularities. Since $F_{1} \simeq\left\{u^{2}+y^{3}=0\right\} \subseteq$ $\mathbb{P}(4,2,2,3)$, we know that $F_{1}$ is an irreducible divisor. We also see that $a\left(F_{1}, V\right)=2 / 3$. Thus, by $(2.2)$, we have

$$
a\left(F_{1}, X\right)=a\left(F_{1}, U,-\frac{1}{2} \operatorname{div}_{U}(y)\right)=2 / 3+1 / 2 \cdot 2 / 3=1 .
$$

LemMA 4.5. Let $Z, U^{\prime}, Q^{\prime}$ be as in (4.3). Let $h_{1}: W_{1} \rightarrow Z$ be the blow up at $Q^{\prime}$ with weight $(x, y, z, u)=\frac{1}{2}(2,1,1,1)$ in $U^{\prime}(\subseteq Z)$. Then the exceptional set $G_{1}$ of $h_{1}$ is an irreducible divisor with $a\left(G_{1}, X\right)=1$, and $W_{1}$ has non terminal singularities.

Proof. By a computation of the blow up, we see that there is an open subset $U^{\prime \prime} \subseteq W_{1}$ (which is the $z$-chart of $W_{1}$ ) such that

$$
U^{\prime \prime} \simeq\left\{\begin{array}{c}
u^{2}+x^{3} z^{2}+\left(g\left(y^{2} z, y z\right) / y^{4} z^{2}\right) \cdot x \\
+h\left(y^{2} z, y z\right) / y^{6} z^{4}=0
\end{array}\right\} / \mathbb{Z}_{2}(1,0,0,1),
$$

which shows that $W_{1}$ has non terminal singularities. We know that $G_{1 \mid U^{\prime \prime}}=$ $\operatorname{div}_{U^{\prime \prime}}\left(u^{2}+y^{2}\right)$ and that $G_{1}$ is an irreducible divisor by considering the $\mathbb{Z}_{2^{-}}$ action on $G_{1}$. We also see that $a\left(G_{1}, Z\right)=1 / 2$. By using $(2.2)$, we get

$$
\begin{aligned}
a\left(G_{1}, X\right) & =a\left(G_{1}, Z,-\frac{1}{3} F-\frac{1}{2} g_{*}^{-1} E\right)=a\left(G_{1}, U^{\prime},-\operatorname{div}_{U^{\prime}}(y)\right) \\
& =1 / 2+1 / 2=1,
\end{aligned}
$$

which completes the proof. 
4.6. Thus we obtain a projective birational morphism $\psi_{1}=h_{1} \circ g \circ f$ : $W_{1} \rightarrow X$ such that the exceptional set of $\psi_{1}$ consists of three prime divisors $\left(h_{1} \circ g\right)_{*}^{-1} E, h_{1 *}^{-1} F$ and $G_{1}$. We also know that $a\left(\left(h_{1} \circ g\right)_{*}^{-1} E, X\right)=1 / 2$ and $a\left(h_{1 *}^{-1} F, X\right)=a\left(G_{1}, X\right)=1$. However $\psi_{1}: W_{1} \rightarrow X$ is not a terminal *-economic resolution since $W_{1}$ has non terminal singularities.

Proposition 4.7. Let $P \in X, Z, \varphi: Z \rightarrow X$ be in (4.1) and (4.3). Then $-K_{Z}$ is $\varphi$-nef.

Proof. Let $D_{X}=\operatorname{div}_{X}(z)$. Then $D_{X} \in\left|-K_{X}\right|$ and has a unique singular point which is a rational double point of type $E_{7}$. Let $D_{Z}=\varphi_{*}^{-1} D_{X}$ be the birational transform of $D_{X}$. Then $\varphi_{\mid D_{Z}}: D_{Z} \rightarrow D_{X}$ is a crepant morphism and $D_{Z} \in\left|-K_{Z}\right|$. Let $C_{1}=g_{*}^{-1} E_{\mid D_{Z}}$ and $C_{2}=F_{\mid D_{Z}}$. These are irreducible curves on $Z$, and we see that $C_{1}$ is a nonsingular rational curve such that $\left(C_{1}^{2}\right)_{D_{Z}}=-2$ and $\left(C_{1} \cdot C_{2}\right)_{D_{Z}}=1$. (There is a rational double point of type $D_{5}$ on $D_{Z}$. This point lies on $C_{2}$ and not on $C_{1}$.)

Let $C$ be an irreducible curve on $Z$ such that $\varphi(C)$ is a point. Then either $C \subset F$ or $C \subset g_{*}^{-1} E$. If $C \subset F$, then $\left(-K_{Z} \cdot C\right)>0$ since $-K_{Z}$ is $g$-ample. If $C \subset g_{*}^{-1} E$ and $C \neq C_{1}$, then $C$ and $D_{Z}$ meets properly, hence $\left(-K_{Z} \cdot C\right)=\left(D_{Z} \cdot C\right) \geq 0$. If $C=C_{1}$, then

$$
\left(-K_{Z} \cdot C\right)=\left(-K_{Z \mid D_{Z}} \cdot C_{1}\right)=\left(\frac{1}{2} C_{1}+C_{2} \cdot C_{1}\right)_{D_{Z}}=1 / 2 \cdot(-2)+1=0 .
$$

Thus we have $\left(-K_{Z} \cdot C\right) \geq 0$ in any cases.

Theorem 4.8. Let $P \in X$ be as in (4.1). Then $P \in X$ has no terminal *-economic resolutions.

Proof. Assuming that there is a terminal *-economic resolution $\psi$ : $W \rightarrow X$, we shall derive a contradiction. The exceptional set of $\psi$ consists of three prime divisors, two of them have discrepancies 1 and one of them has discrepancy $1 / 2$ over $P(\in X)$. We also know that $\rho(W / X)=3$. By (2.4), $K_{W}$ is not $\psi$-nef, hence there is an extremal ray $R$ on $W$ such that $\left(K_{W} \cdot R\right)<0$. We apply the Minimal Model Program over $X$ starting with the contraction of $R$. Then, after a finite number of flips $W \rightarrow W^{\prime}$, we get to a divisorial contraction $h^{\prime}: W^{\prime} \rightarrow Z^{\prime}$. Since $\rho\left(Z^{\prime} / X\right)=2$, we can continue the Minimal Model Program. There are several flips $Z^{\prime} \rightarrow-\rightarrow Z^{\prime \prime}$ and a divisorial contraction $g^{\prime \prime}: Z^{\prime \prime} \rightarrow Y^{\prime \prime}$. Since $\rho\left(Y^{\prime \prime} / X\right)=1$, the morphism $f^{\prime \prime}: Y^{\prime \prime} \rightarrow X$ is a divisorial contraction which contracts one of the prime divisors with discrepancies $\leq 1$ to a point $P$, hence it is isomorphic to 
$f: Y \rightarrow X$ in (4.1) by using (2.3) and (4.2). Since $g^{\prime \prime}: Z^{\prime \prime} \rightarrow Y^{\prime \prime}$ contracts a prime divisor which has discrepancy 1 , it follows from (2.3) and (4.4) that $g^{\prime \prime}: Z^{\prime \prime} \rightarrow Y^{\prime \prime}$ is isomorphic to $g: Z \rightarrow Y$ in (4.3). Since $-K_{Z}$ is $\varphi$-nef, there are no flipped curves on $Z$. Therefore $Z^{\prime}$ and $Z^{\prime \prime}(\simeq Z)$ are isomorphic. Thus, by (2.3), we see that $h^{\prime}: W^{\prime} \rightarrow Z^{\prime}$ is isomorphic to $h_{1}: W_{1} \rightarrow Z$ in (4.5). This is a contradiction by comparing the singularities of $W^{\prime}$ with those of $W_{1}$.

\section{$\S 5$. Canonical economic and canonical $*$-economic resolutions}

In this section, we shall study canonical economic and canonical $*$ economic resolutions, and prove that every 3-dimensional terminal singularity has such resolutions. The proof here is an easy application of [FA92, 17.10].

Theorem 5.1. Let $P \in X$ be a germ of a 3-dimensional terminal singularity. Then $P \in X$ admits a canonical economic and a canonical *-economic resolutions.

Proof. By [Reid87, (6.4)], a general member $D_{X} \in\left|-K_{X}\right|$ has only rational double point at $P$. Let $\mathcal{H}_{X} \subseteq\left|-K_{X}\right|$ be a movable linear system on $X$ such that a general member of $\mathcal{H}_{X}$ has a rational double point at $P$. Then, by [Alex94, 1.21], we have $a\left(E, X, \mathcal{H}_{X}\right) \geq 0$ for all prime divisors $E$ over $X$, which means that $K_{X}+\mathcal{H}_{X}$ is canonical. Let $f: Y \rightarrow X$ be a resolution of singularities of $X$ such that the exceptional locus of $f$ is a divisor and that the birational transform $\mathcal{H}_{Y}=f_{*}^{-1} \mathcal{H}_{X}$ is free. Then $a\left(F, Y, \mathcal{H}_{Y}\right)>0$ for all prime divisors $F$ over $Y$, hence $K_{Y}+\mathcal{H}_{Y}$ is terminal. Let $\mathcal{E}(f)$ be the set of all exceptional prime divisors of $f$.

Claim 5.2. For a prime divisor $E$ over $P(\in X)$, we have the following:

(1) If $a(E, X) \leq 1$, then $a\left(E, X, \mathcal{H}_{X}\right)=0$.

(2) If $a\left(E, X, \mathcal{H}_{X}\right)=0$, then $E \in \mathcal{E}(f)$.

Proof. Since $K_{Y}+\mathcal{H}_{Y}$ is terminal, we see that $a\left(E, X, \mathcal{H}_{X}\right)=0$ implies $E \in \mathcal{E}(f)$. Since $K_{X}+\mathcal{H}_{X}$ is canonical and $\mathcal{H}_{X} \subseteq\left|-K_{X}\right|$, we see that $a\left(E, X, \mathcal{H}_{X}\right) \neq 0$ implies $a\left(E, X, \mathcal{H}_{X}\right) \geq 1$. We know that $P$ is in the base locus of $\mathcal{H}_{X}$, hence we have $a(E, X)>a\left(E, X, \mathcal{H}_{X}\right) \geq 1$. Therefore $a(E, X) \leq 1$ implies $a\left(E, X, \mathcal{H}_{X}\right)=0$. 
Now we return to the proof of (5.1). Let $\mathcal{E}_{1}=\{E \in \mathcal{E}(f) \mid a(E, X)<1\}$ and let $\mathcal{E}_{2}=\{E \in \mathcal{E}(f) \mid a(E, X) \leq 1\}$. By $(5.2), \mathcal{E}_{1}$ (resp. $\mathcal{E}_{2}$ ) is the set of all exceptional prime divisors $E$ with $a(E, X)<1$ (resp. $a(E, X) \leq 1$ ), and each element $E \in \mathcal{E}_{i}(i=1,2)$ satisfies $a\left(E, X, \mathcal{H}_{X}\right)=0$. Hence we can apply $\left[\right.$ FA92, 17.10] and get a normal $\mathbb{Q}$-factorial 3-fold $X\left(\mathcal{E}_{i}\right)$, a birational map $h_{i}: Y \rightarrow X\left(\mathcal{E}_{i}\right)$ and a birational morphism $g_{i}: X\left(\mathcal{E}_{i}\right) \rightarrow X(i=1,2)$ such that

(i) $f=g_{i} \circ h_{i}$,

(ii) $K_{X\left(\mathcal{E}_{i}\right)}+g_{i *}^{-1} \mathcal{H}_{X}=g_{i}^{*}\left(K_{X}+\mathcal{H}_{X}\right)$, and

(iii) $h_{i}$ is isomorphic at each generic point of $E \in \mathcal{E}_{i}$, and contracts each $E^{\prime} \in \mathcal{E}(f) \backslash \mathcal{E}_{i}$.

By (ii), we know that $K_{X\left(\mathcal{E}_{i}\right)}+g_{i *}^{-1} \mathcal{H}_{X}$ is canonical, in particular $X\left(\mathcal{E}_{i}\right)$ has only canonical singularities. We also see that $\mathcal{E}\left(g_{i}\right)=\mathcal{E}_{i}$ by (iii). Therefore $g_{1}: X\left(\mathcal{E}_{1}\right) \rightarrow X$ and $g_{2}: X\left(\mathcal{E}_{2}\right) \rightarrow X$ are a canonical economic and a canonical $*$-economic resolutions respectively.

\section{REFERENCES}

[Alex94] V. Alexeev, General elephants of $\mathbb{Q}$-Fano 3-folds, Compositio Math., 91 (1994), 91-116.

[Art68] M. Artin, On the solutions of analytic equations, Invent. Math., 5 (1968), 277-291.

[Art69] - Algebraic approximation of structures over complete local rings, Publ. Math. I.H.E.S., 36 (1969), 23-58.

[Hay99] T. Hayakawa, Blowing ups of 3-dimensional terminal singularities, Publ. RIMS, Kyoto Univ., 35 (1999), 515-570.

[Hay00] Blowing ups of 3-dimensional terminal singularities, II, Publ. RIMS, Kyoto Univ., 36 (2000), 423-456.

[Hay05] Gorenstein resolutions of 3-dimensional terminal singularities, Nagoya Math. J., 178 (2005), 63-115.

[Kaw84] Y. Kawamata, The cone of curves of algebraic varieties, Ann. of Math., 119 (1984), 603-633.

[FA92] J. Kollár et al., Flips and abundance for algebraic threefolds, Astérisque, 211 (1992).

[KM98] J. Kollár and S. Mori, Birational geometry of algebraic varieties, Cambridge University Press, 1998.

[Reid80] M. Reid, Canonical threefolds, Géométrie Algébrique Angers (A. Beauville, ed.), Sijthoff \& Noordhoff (1980), pp. 273-310.

[Reid83] — Minimal models of canonical threefolds, Algebraic Varieties and Analytic Varieties, Adv. Stud. Pure Math. 1, Kinokuniya and North-Holland (1983), pp. 131-180. 
[Reid87] — Young person's guide to canonical singularities, Algebraic Geometry, Bowdoin 1985, Proc. Symp. Pure Math., 46 (1987), pp. 345-416.

Department of Mathematics

Kanazawa University

Kakuma-machi

Kanazawa, 920-1192

Japan

thaya@kenroku.kanazawa-u.ac.jp 